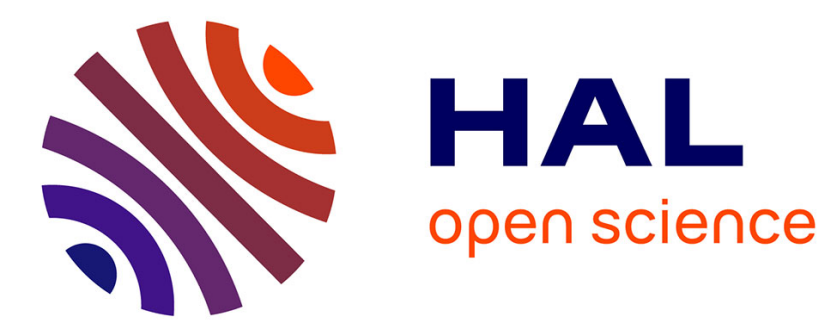

\title{
Micro-emboli detection: an ultrasound Doppler signal processing viewpoint
}

Jean Marc Girault, Denis Kouamé, Abdeldjalil Ouahabi, Frédéric Patat

\section{To cite this version:}

Jean Marc Girault, Denis Kouamé, Abdeldjalil Ouahabi, Frédéric Patat. Micro-emboli detection: an ultrasound Doppler signal processing viewpoint. IEEE Transactions on Biomedical Engineering, 2000, 47 (11), pp.1431 - 1439. 10.1109/10.880094 . hal-01076489

\section{HAL Id: hal-01076489 \\ https://hal.science/hal-01076489}

Submitted on 22 Oct 2014

HAL is a multi-disciplinary open access archive for the deposit and dissemination of scientific research documents, whether they are published or not. The documents may come from teaching and research institutions in France or abroad, or from public or private research centers.
L'archive ouverte pluridisciplinaire HAL, est destinée au dépôt et à la diffusion de documents scientifiques de niveau recherche, publiés ou non, émanant des établissements d'enseignement et de recherche français ou étrangers, des laboratoires publics ou privés. 


\title{
Micro-emboli detection: an ultrasound
}

\section{Doppler signal processing view point}

\author{
Jean-Marc Girault, Student Member, IEEE, Denis Kouamé, Member, IEEE,
} Abdeldjalil Ouahabi, Member, IEEE, and Frédéric Patat

\begin{abstract}
Several studies have been carried out in the last twenty years on the characterization and detection of cerebral artery emboli. From the detection point of view, the existing methods are in a large part based on the classical Fourier analysis of which the well known limitations yield poor accuracy. This paper first recalls existing methods based on Fourier, Wigner-Ville and wavelet approaches.

Then, it presents new emboli detection methods based on parametric signal processing approaches. The basic idea of this parametric method is to track the deviation between the Doppler embolic signal and its modeled signal. It uses an autoregressive model of the Doppler signal to construct a decision information which contains the signature of the sought micro-embolus. Then, the sought embolus is detected with a certain probability of truth.

In order to perform a comparison between these new methods and classical analysis, a realistic embolic signal simulation is also presented. Furthermore, to validate our theoretical study, we test new algorithms with in vivo signals. This comparison emphasizes the significant inaccuracy of existing methods as far as micro-emboli detection is concerned. Finally in vivo investigations are performed to validate the theoretical study.
\end{abstract}

\section{Keywords}

Autoregressive, Decision Information, Detection, Emboli, Non-Parametric, Parametric.

\section{INTRODUCTION}

$I^{2}$ recent years, researchers have taken interest in monitoring clinically silent circulating microemboli in the hope to identify patients at increased risk of thromboembolic stroke.

The authors are with the LUSSI/GIP ULtrasons, Tours, France. E-mail: jmgirault@univ-tours.fr.

This work was sponsored by the Région Centre of France. 
Embolism, which corresponds to an intravascular migration of an insoluble body, can stop the blood flow and lead to ischemia.

Obstruction of a blood vessel can be performed either by a large embolus (Fig. 1) or a hail of small emboli. Emboli may appear in the shape of a gas bubble [1], a fat globule [2], a blood clot, a plaque of atheroma and a piece of thrombus [3], [4], [5].

Concerning micro-emboli, on the one hand their origin and their processes of development remain fairly unknown notably because of their small size and the limitations of detection systems; on the other hand circulating micro-emboli may indicate an increased risk of macro-embolism leading to stroke.

The scientific community agrees that transcranial Doppler system [6] is adapted for embolic research in the cerebral circulation.

Nowadays, manufacturers have developed commercial software systems aimed at automatically detecting, counting and characterizing particles in human blood. But these systems do not permit to point out all emboli and particularly the smallest.

From a signal processing standpoint, most of the available techniques in automatic emboli detection are based on the power spectrum obtained by classical Fourier analysis, the limitation of which are well-known (conflict between time and frequency resolutions, choice of analyzing windows).

Different alternatives can be envisaged. To our knowledge, they are all non parametric techniques. The first one is the Wigner-Ville transform [7] which gives a better temporal resolution and then improves the detection. Another technique, recently introduced by Guetbi and al [8], Lui and al [9], is the wavelet analysis. These techniques provide better results than those obtained by the Fourier analysis.

The motivation of our paper is to propose a new automatic detection method based on an abrupt change approach in order to investigate the problem of micro-emboli detection. This original approach consists in highlighting discontinuities which appear in stationary segments of the signal (segmentation) by calculating a decision information (DI). DI is a quantity generated from the Doppler signal which 
contains the embolus signature. Detecting an embolus comes down to doing an hypothesis test that explains the fact that there is or there is not an embolus, respectively. To construct the decision information, we do not directly use the Doppler signal but a parametric model of this signal. This parametric model is a time-variant AutoRegressive (AR) model. Here, we propose to use two DI: the autocorrelation of the innovation [10] and the modulus of the derivative of AR parameters.

In order to compare the presented methods, we propose to apply them to a simulated embolic Doppler signal. These artificial signals are performed with an algorithm based on simulations proposed by Wendling [11]. The comparison between methods is performed using the Receiver Operating Characteristic (ROC) curves. These curves permit to highlight the performances of each method in terms of probability of non detection and probability of false alarm. Finally an in vivo validation is provided.

This paper is organized as follows: first we describe a realistic embolic Doppler signal simulation which allows us to test the presented methods. Finally, the performances of the methods are evaluated and discussed using ROC curves.

\section{Simulation of the Doppler embolic ultrasound Signal}

In order to compare the different methods presented in the following chapters, we develop simulations of Doppler embolic signals. These synthetic signals, that are very realistic, offer a better flexibility than using real signals.

\section{A. Problem statement}

Embolic Doppler signals observed can be described by a random process $x(t)$ such that:

$$
x(t)=S(t)+\sum_{i=1}^{n e} E_{i}\left(t-\theta_{e i}\right)+\sum_{k=1}^{n a} A_{k}\left(t-\theta_{a k}\right)+B(t) .
$$

This relation describes the relevant information that constitutes the embolic Doppler signal. $S(t)$, the background signal backscattered by the blood, may be considered as a piece-wise stationary signal; for 
each $i, E_{i}$ represents a high intensity signal of brief duration, with time occurrence $\theta_{e i}$, and corresponds to the temporal signature of an embolus; the $A_{k}$ terms may be related to artifacts occurring at times $\theta_{a k}$; finally, measurement noise that is stationary throughout the observation is gathered in the term $B(t)$; the entities ne and na represent respectively the random number of temporal occurrences of brief useful events and artifact transient signal over the period of observation. Notice that $S(t)$ and $E_{i}$ are related because, in blood vessels, we suppose that emboli circulate with the same speed as Red Blood Cells (RBC), haemodynamically this looks like a good approximation.

In our study, we do not take into account the artifact term because the technique proposed by Smith and al [12], based on a dual gated Doppler ultrasound, allows us to differentiate between emboli (unidirectional signals) and artifacts signals (bi-directional signals). Usually, the Doppler signal to measurement noise ratio is about $15 \mathrm{~dB}$, without emboli. Now let us focus on expressing $S(t)$ and $E(t)$.

\section{B. Modeling of Doppler embolic signal}

The original simulation of the Doppler embolic signal, that we propose, is based on the temporal simulation of the pulsed Doppler signal [11], in which we have incorporated an embolus. However, the numerical implementation of this algorithm does not depend on the aspect of the embolus. More precisely, only the energy backscattered by the embolus embedded in the circulating blood is taken into account.

In fact, the Doppler embolic signal must include random characteristics due to the random phase of scattering particles which are present in the sample volume and must take into account the fact that an embolus crosses a multi-scatterer medium. Other effects such as geometric broadening and spatially varying velocity also affect the signal.

The radio-frequency backscattered signal from a single particle within the sample volume is given 
by:

$$
R_{j}(t)=C_{j} \sigma_{R B C j} \cos \left(\psi_{d}(t)+\phi_{j}\right)
$$

with

$$
\psi_{d}(t)=\omega_{0} t+\omega_{d}(t)
$$

and with

$$
\omega_{d}(t)=\frac{2 \omega_{0}}{c} \cos (\alpha) \int_{0}^{t} v(\tau) d \tau
$$

$C_{j}$ both is proportional to the amplitude of the two-way beam pattern at the location of the particle $j$ and to the envelope of the transmitted pulse and also to the shape of the receiver gate. $\sigma_{R B C j}$ is the backscattering cross section of one particle. Owing to the random location of the particle $j$, the phase $\phi_{j}$ is random and uniformly distributed. Finally, $\psi_{d}(t)$ is the time-dependent phase caused by the particle motion. $\omega_{d}(t)$ is the Doppler shift related to the instantaneous flow velocity $v(t)$, to the ultrasound speed $c$ and to the carried frequency $\omega_{0} . \alpha$ is the angle between the direction of the ultrasound beam and the blood flow. The total signal from all scatterers is obtained by summing over all particles $j$. In the Doppler device, this radio-frequency signal is multiplied by $\cos \left(\omega_{0} t\right)$ and low pass filtered in order to get the Doppler audio-signal of which the first quadrature has the form:

$$
S(t)=\sum_{j} C_{j} \sigma_{R B C j} \cos \left(\omega_{d}(t)+\phi_{j}\right)
$$

The embolic signature is expressed as:

$$
E_{i}(t)=C_{i} \sigma_{E i} \cos \left(\omega_{d}(t)+\phi_{i}\right)
$$

where $\sigma_{E i}$ is the backscattering cross section of one embolus. In order to present the problem in a simpler manner, suppose that there is only one embolus in the sample volume (Fig. 1) . Here, we consider that an embolus is a large RBC; that is not restrictive. In practice, we measure "the embolus to blood ratio" (EBR) already introduced by Moehring and Klepper [13]: 


$$
E B R=10 \log _{10}\left(\frac{P_{E+B}}{P_{B}}\right)
$$

$P_{E+B}$ is the backscattered power measured with blood and embolus in the Doppler sample volume, $P_{B}$ is the backscattered power measured from blood alone in the sample volume (Fig. 2). Figs. 2 and 3(a) show that artificial embolic signals are very realistic compared to those recorded from a classical Doppler device. Those signals are obtained with a $2 \mathrm{MHz}$ emitted frequency and a $2 \mathrm{~mm}$ sample volume length. In this study, sets of $3,6,8$ and $10 d B$ have been simulated. In the following chapters, all illustrated examples are performed with $E B R=6 d B$.

\section{Non-PARAMETR.tC Methods}

As it is introduced by Spencer [14], the embolic Doppler signal is a signal in which sharp transients appear. In order to identify irregularities (emboli) in the signal backscattered by the blood, it is common to divide long-term signals into blocks of short duration and to compute a decision information (DI). DI is a quantity generated from the Doppler signal which contains the embolus signature. In all the methods, the detection is obtained if $D I \geq T_{\text {hresh }}$, where $T_{h r e s h}$ is the detection threshold.

\section{A. The Spectrogram or the Short Time Fourier Transform (STFT)}

The basic idea of the spectrogram is to find the power spectral density of the signal at time $t$ by analyzing a small segment of the signal around $t$. It is written as follows:

$$
P(t, f)=\left|\int_{-\infty}^{+\infty} x(\tau) g^{*}(\tau-t) e^{-j 2 \pi f \tau} d \tau\right|^{2}
$$

Here, $x(t)$ represents the analyzed Doppler signal. The signal is multiplied by a window function $g$ in order to reduce the random effects resulting from the computation of $P(t, f)$. The superscript asterisk denotes complex conjugation. The spectrogram (see Fig. 2(b)) describes approximately the energy density of the signal at point $(t, f)$. The problem with the STFT is that both time and frequency resolutions of the transform are fixed over the entire time-frequency plane. In addition, choosing a 
short analysis window may cause poor frequency resolution. On the other hand, while a long analysis window may improve frequency resolution, it compromises the assumption of stationarity within the window. In this study, the STFT is performed using a 64 points Hamming window with an overlap of $50 \%$ and no zero padding is used. The decision information (DI) is the same as in commercial devices, it is the maximum of the power spectrum:

$$
D I_{1}=\left.\max (P(t, f))\right|_{t}
$$

where subscript $t$ denotes that the maximum power spectrum is sought for each time $t$ and for all frequencies. Figs. $4(\mathrm{a})$ and $4(\mathrm{~d})$ show $D I_{1}$ with $(E B R=6 d B)$ and without an embolus crossing the sample volume. These figures indicate that a high intensity associated to an embolus appears with a poor resolution.

\section{B. The Wigner-Ville Transform (WVT)}

The Wigner-Ville distribution is a useful tool suitable for the analysis of time-varying signals [15], [16]. The main advantage of the WVT is that it does not require the analyzed signal to be stationary within the analysis time interval. It produces results with high temporal resolution.

Recently, Smith and al [7] have introduced the use of the WVT (Fig. 3(a)) for the analysis of embolic signals. They emphasize that the use of the WVT gives better results than the Fourier approach in terms of emboli detection.

The WVT of a signal $x(t)$ is defined as:

$$
W(t, f)=\int_{-\infty}^{+\infty} x\left(t+\frac{\tau}{2}\right) x^{*}\left(t-\frac{\tau}{2}\right) e^{-j 2 \pi f \tau} d \tau
$$

Here, the commonly used DI is the maximum energy of $W(t, f)$.

$$
D I_{2}=\left.\max (W(t, f))\right|_{t}
$$

where subscript $t$ denotes that the maximum of $W(t, f)$ is performed at each time $t$ and for all frequencies. Figs. $4(\mathrm{~b})$ and $4(\mathrm{e})$ show $D I_{2}$ with $(E B R=6 d B)$ and without an embolus. 


\section{The Wavelet Transform (WT)}

The wavelet theory was tied together by Grossman and Morlet in 1984 [17]. Later a preliminary study applied to emboli detection was proposed in our research group by Guetbi and al [8]. The wavelet transform of a signal $x(t)$ is the decomposition of this signal over a set of functions obtained after dilatation and translation of an analyzing wavelet $\zeta$ which verifies some admissibility conditions [17]. The coefficients or details resulting from this decomposition are denoted $C_{a, b}$ with:

$$
C_{a, b}=\int_{0}^{+\infty} x(t) \zeta_{a b}^{*}(t) d t
$$

where $a$ and $b$ respectively represent the scaling factor (dilatation/compression coefficient) and the time (shifting coefficient) and the superscript asterisk denotes the complex conjugation. The set of basic wavelets is expressed by:

$$
\zeta_{a b}(t)=\frac{1}{\sqrt{|a|}} \zeta\left(\frac{t-b}{a}\right),
$$

for $a \neq 0, b \in \Re$, where $\Re$ denotes the real set. The set of $C_{a, b}$, constitutes the wavelet transform.

Here we use the Morlet wavelet:

$$
\zeta(t)=e^{j \omega t} e^{-\frac{t^{2}}{2}}
$$

The Morlet wavelet is chosen first because of its simplicity and secondly because its shape looks like the embolus signature. WT has a variable time-frequency resolution over the time-frequency plane by providing good time resolution at high frequency and good frequency resolution at low frequencies. The detection of an embolus is given when the maximum of $C_{a, b}$ crosses the threshold. Here, the DI is given by:

$$
D I_{3}=\left.\max \left(C_{a, b}\right)\right|_{b},
$$

where subscript $b$ denotes that the maximum of $C_{a, b}$ is evaluated at each shifting coefficient $b$ and for all scaling factors $a$. Figs. $4(\mathrm{c})$ and $4(\mathrm{f})$ show $D I_{3}$ with $(E B R=6 d B)$ and without an embolic signal. 


\section{Detection Through Parametric Modeling}

The presented approach is in keeping with the detection of abrupt change [18], [19]. This approach consists in pointing out discontinuities existing in stationary segments of signal by computing the DI. To built the DI, a parametric estimation based on time-variant AutoRegressive (AR) modeling is used.

\section{A. Time-Variant AR Modeling}

The parametric analysis consists in modeling the Doppler signal as the output of a linear filter driven by a white noise. This filter, referred to as AR, is a linear combination of the previous samples (Regressive) of the output itself (Auto). For more details, we can refer to [20] and [21] for example:

$$
x(n)=-\sum_{i=1}^{i=p} a_{i}(n) x(n-i)+\eta(n),
$$

where $\eta$ is a white complex noise and $n$ is the sample time. An order test [22] shows that $p=2$ may be sufficient.

There are many algorithms to compute AR parameters, here we focus on the most popular one: the adaptive least-square algorithm. Note that if the background noise added to the signal is colored, an instrumental variable (IV) algorithm [23] should rather be used.

Let us rewrite equation (11) in a matrix form:

$$
x(n)=\phi_{n}^{T} \theta+\eta(n),
$$

with

$$
\begin{gathered}
\phi_{n}^{T}=[-x(n-1),-x(n-2)], \\
\theta=\left[a_{1}, a_{2}\right]^{T^{T}} .
\end{gathered}
$$

If long data sequences are available, it is possible to use a time-variant identification method. In this section, we describe a time-variant algorithm that allows us to obtain a new set of parameters whenever a new sample is available. This is accomplished by updating the previously evaluated parameter using 
the prediction error, and weighting by means of a forgetting factor $\lambda$. In fact, using a forgetting factor is equivalent to the introduction of an exponential window. The length of this window indicates which of the last samples are effectively taken into account to compute AR parameters. For example, if the forgetting factor is equal to 0.95 , the 20 last samples are mainly accounted for in the window, whereas if it is equal to 0.99 the last 100 samples are taken into account.

This adaptive least-squares method evaluates the $a_{i}$ parameters by minimizing the cost function:

$$
J_{k}=\sum_{n=1}^{k} \lambda^{k-n}(x(n)-\hat{x}(n))^{2},
$$

where in practice $0.80<\lambda<1$ and $k$ is the index of the last sample considered.

The general expression of the recursive adaptive algorithm using (12) is given by [21] and [24]:

$$
\begin{gathered}
P_{n}=\frac{P_{n-1}}{\lambda}\left(1-\frac{P_{n-1} \phi_{n} \phi_{n}^{I^{\prime}} P_{n-1}}{\lambda+\phi_{n}^{T} P_{n-1} \phi_{n}}\right), \\
\epsilon_{n}=x_{n}-\phi_{n}^{T} \hat{\theta}_{n-1}, \\
\hat{\theta}_{n}=\hat{\theta}_{n-1}+P_{n} \phi_{n} \epsilon_{n},
\end{gathered}
$$

where $\hat{\theta}_{n}$ is the estimated parameters vector, $P_{n}$ is the gain, $\phi_{n}$ is the observation vector and $\epsilon_{n}$ is the prediction error.

Due to the recursive nature of this algorithm, first a transient region appears and secondly it is required to initialize the gain $P_{n}$ and the AR parameters. A standard initialization value of the gain is $P_{0}=10 I$ ( $I$ is an identity matrix).

The underlying idea is that a parametric model of the Doppler signal without emboli is no longer valid when an embolus is present in the sample volume. In this case, the embolus signature appears in the prediction error (Fig. 5(c)) as well as in AR parameters (Fig. 6(b)). 


\section{B. Parametric decision information}

- The first decision information, presented by Kouamé and al [25] with a differential approach, is the autocorrelation of the prediction error $\epsilon(n)$ recursively estimated at a time different to the initial time. The prediction error or the innovation can be rewritten as follows:

$$
\epsilon(n)=x(n)-\hat{x}(n) .
$$

Its autocorrelation is given by:

$$
\Gamma_{\epsilon \epsilon}(n)=\sum_{m=-\infty}^{m=+\infty} \epsilon(m) \epsilon^{*}(m-n)
$$

Here the autocorrelation is the decision information. $\Gamma_{\epsilon \epsilon}(n)$ is computed for a time $(n=1)$ that is just after the initial $(n=0)$. Indeed, without an embolus, the innovation tends to be a white noise and its autocorrelation is quasi zero at any time different to the initial. From (15) the autocorrelation can be recursively estimated as in [25], [26]:

$$
D I_{4}(n)=\gamma D I_{4}(n-1)+(1-\gamma) \epsilon(n) \epsilon(n-1),
$$

$\gamma=0.9$ is a weighting factor.

- The main idea of the second DI is that an abrupt change produced by an embolus is a high frequency phenomenon. To increase the contrast between an embolus and the blood, a high pass filter (or a derivative operation) can be used. Accordingly, we propose a DI based on the modulus of the AR parameters derivative. This latter is based on the idea introduced by Hagglund [27] and Girault [28] :

$$
D I(t)=\frac{\left|d \hat{a}_{1}(t)\right|}{d t} .
$$

From a numerical point of view, the DI becomes:

$$
D I_{5}(n)=\left|\hat{a}_{1}(n)-\hat{a}_{1}(n-1)\right|
$$

In order to reduce the false alarm, we can introduce a weighting factor, $\gamma$ :

$$
D I_{5}(n)=\gamma D I_{5}(n-1)+(1-\gamma)\left|\hat{a}_{1}(n)-\hat{a}_{1}(n-1)\right| .
$$


Fig. 6 shows the evolution of $a_{1}(n)$ with $(E B R=6 d B)$ and without an embolic signal. These curves obtained with a forgetting factor of 0.8 explain the stochastic nature of both the AR parameters and their decision information. Indeed, the forgetting factor permits us to obtain a trade-off between a good accuracy and reduced statistical fluctuations on parameters estimation. Fig. 7 illustrates the DI of parametric methods with $(E B R=6 d B)$ and without an embolic signal. At this stage, it seems to be a promising way to detect emboli.

\section{Comparison of the Presented Methods on Simulated Signals}

In order to compare the previous methods, four sets of simulated data have been computed (each containing 100 Doppler embolic signals). These four sets correspond to different values of $E B R: 3,6,8$ and $10 d B$.

\section{A. Case of only one embolus}

The previous methods are compared using different statistic tools.

- Non detection is the fact of not detecting an embolus which has effectively crossed the sample volume. We evaluate the probability of non detection (PND) by:

$$
P N D=\frac{N N D}{N R}
$$

where $N N D$ is number of non detections obtained for $N R$ realizations.

- False alarm is the fact of detecting an embolus event where in reality no embolus has crossed the sample volume. We evaluate the probability of false alarm (PFA) by:

$$
P F A=\frac{N F A}{N R}
$$

where $N F A$ is number of false alarms obtained for $N R$ realizations.

For example, from the Fig. 8(d), a test is performed in order to know if there is an embolus. Knowing that an embolus is present in the sample volume, if there is more than one value of $D I_{1}$ superior to $T_{\text {hresh }}$ then there is a false alarm. In the case where there is only one value superior to the threshold, 
and located in the embolus region, there is a true detection. These operations are performed 100 times in order to evaluate the probabilities for each threshold and for each value of EBR.

- The Receiver Operating Characteristic (ROC) curves illustrate the evolution of the PFA versus the PND. It passes through the point $(1,0)$ and $(0,1)$, it is convex and under the first bisecting line. An ideal method is a method that approaches the point $(0,0)$, i.e. $P F A=P N D=0$. An example is illustrated in the Fig. 8(a).

Curves of Figs. 9(a) and 9(b) show the PFA versus the PND in the Fourier $\left(D I_{1}\right)$, Wigner-Ville $\left(D I_{2}\right)$ and Wavelet $\left(D I_{3}\right)$ approaches, respectively. When no embolus is detected, the curve tends to a straight line that passes through the point $(1,0)$ and $(0,1)$. Each plotted numbers correspond to a fixed energy level such as $E B R(3,6,8,10 d B)$. Figs. 9(a) and 9(b) illustrate the superiority of the Wigner-Ville and Wavelet approaches compared to the Fourier approach. Indeed, for Doppler embolic signals with a fixed energy, curves obtained by the WVT and the WT are very close to the ideal point $(0,0)$; this is not verified for the Fourier approach. This can be explained by the good behavior of the WVT and WT in terms of time and frequency resolutions. Fig. 9(b) emphasizes the fact that the WVT gives same results as the WT, but the computation speed of the WT is higher.

Now, let us focus our attention on the comparison between non parametric and parametric approaches. Fig. 10(a) highlights that the parametric method based on the derivative of AR parameters $\left(D I_{5}\right)$ is by far superior to all non parametric methods. We can explain the good results obtained from parametric methods because they use supplementary information that it is not taken into account in all non parametric methods. This complementary information is the linear prediction error. When an embolus crosses the sample volume, this random event, not predictable by nature, implies an abrupt change in the prediction of the signal. This predictive information combined with the energetic information explains in part the superiority of the parametric methods.

Concerning the comparison between parametric methods, it seems that the one based on the autocorrelation of the prediction error $\left(D I_{4}\right)$ gives, by far, better results than the one related to the 
derivative of $\mathrm{AR}$ parameters. This is due to the noise introduced by numerical derivation.

In practice, it is reasonable to consider that $P F A=P N D \leq 10 \%$ produces reliable emboli detection. In these conditions, we apply the same criterion to simulated data and we search, on ROC curves (see Fig.s 9, 10), which methods that are located in the area delimited by $P F A=P N D \leq 10 \%$. For example, from Fig. 9, it appears that Fourier method can be located in this region only for $E B R \geq 12 d B$. Wigner-Ville and Wavelet approaches are located in this area for $E B R \geq 10 d B$. Parametric methods are located in this area for $E B R \geq 5 \mathrm{~dB}$. A classification of those methods is summarized in Table I. When micro-emboli $(5 d B \leq E B R \leq 10 d B)$ are present, the Fourier approach seems unable to detect them in a reliable manner. However, it seems that parametric approaches will allow their detection (with a good degree of accuracy).

An extrapolation leads us to believe that detection with classical devices is reliable only for $E B R \geq$ $12 d B$. Note that those $E B R$ formulations differ from those provided by [29].

\section{B. Case of two emboli}

In the case of two emboli, it is clear that if two emboli are very close together only high time resolution methods could detect the two separately. This is the case with the Wigner-Ville and the Wavelet approaches. In the Fourier approach, it is possible to detect these two emboli only if the delay between them is higher than the time resolution. Concerning the implemented parametric methods, the detection of both will be possible only if the delay between them is superior to the convergence time (due to the recursive nature of the time-variant method). This convergence time, observed in Fig. 7 can be significantly reduced by the use of a differential operator introduced by [30].

\section{In vivo STUDY}

In order to confirm and validate our theoretical study, we have performed a short in vivo study. From one patient, we have recorded 418 Doppler signals with the MDX-4 TCD-8 DWL Elektronische Systeme GmbH during one hour. Conditions of recording are the following: the emitted frequency is 
$2 \mathrm{MHz}$, the pulse repetition frequency is $6 \mathrm{KHz}$, the sample volume is $2 \mathrm{~mm}$, the depth of analyze is $59 \mathrm{~mm}$ and the insonified vessel is the medium carotid artery.

From the recorded data we have made an energetic classification (according to the $E B R$ ) in order to evaluate the corresponding $R O C$ curves. In the case of high energy all methods have well detected high intensity transient signal (HITS). Concerning low energy, results are illustrated on the Fig. 11. These $R O C$ curves confirm and validate that parametric approaches are better than non-parametric methods. Furthermore Fig. 11 highlights that high resolution methods (Wigner-Ville and Wavelets) are better than the Fourier approach.

\section{Conclusion And Perspectives}

In this paper, we have reviewed the existing methods to detect emboli. Furthermore, we have proposed an algorithm to make artificial Doppler embolic signals. From these synthetic signals, we have compared non parametric methods to new parametric methods with statistical tools such as ROC curves.

From simulations and in vivo signals, this study highlights that the proposed parametric methods are by far better than the existing methods such as the Fourier, Wigner-Ville and Wavelet approaches.

It has been stated that parametric methods have a good ability to detect emboli.

This study leads us to believe that, firstly commercial devices are unable to detect small emboli and secondly that the use of parametric methods is a promising way to detect micro-emboli.

\section{ACKNOWLEDGMENTS}

We gratefully acknowledge Frédéric Salez, Marie-Cécile Bonithon of the Bretonneau Hospital of Tours for their help concerning the recording of Doppler embolic signals and Valérie Labat for her critical comments. 


\section{R.EFERENCES}

[1] M.P. Spencer, S.D. Campbell, J.L. Sealey, F.C. Henry, and J. Lindbergh, "Experiments on decompression bubbles in the circulation using ultrasonic and electromagnetic flowmeters," J. Occup. Med., vol. 11, pp. 238-244, 1969.

[2] G.L. Kelly L, G. Dodi, and B. Eisman, "Ultrasound detection of fat emboli," Surg. Forum, vol. 23, pp. 459-461, 1972.

[3] M.P. Spencer, G.I. Thomas, S.C. Nicholls, and L.R. Sauvage, "Detection of middle cerebral artery emboli during carotid endarterectomy using transcranial doppler ultrasonography," Stroke, vol. 21, pp. 415-423, 1990.

[4] M. Siebler, M. Sitzer, and H. Steinmetz, "Detection of intracranial emboli in patient with symptomatic extracranial carotid artery disease," Stroke, vol. 23, pp. 1652-1654, 1992.

[5] D. Russell, K.P. Madden, W.M. Clark, P. Morten Sandset, and J.A. Zivin, "Detection of arterial emboli using doppler ultrasound in rabbits," Stroke, vol. 22, pp. 253-258, 91.

[6] D.W. Newell and R. Aaslid, Transcranial Doppler, Raven Press Ltd New York, 1992.

[7] J.L. Smith, D.H. Evans, L. Fan, A.J. Thrush, and A.R. Naylor, "Processing doppler ultrasound signals from blood-borne emboli," Ultrasound Med. Biol., vol. 20, pp. 455-462, 1994.

[8] C. Guetbi, D. Kouamé, A. Ouahabi, and J.P. Remenieras, "New emboli detection methods," in Proc. Ultrasonic Symposium, 1997.

[9] P.W. Lui, B.C. Chan, F.H. Chan, P.W. Poon, H. Wang, and F.K. Lam, "Wavelet analysis of embolic heart sound detected by precordial doppler ultrasound during continuous venous air embolism in dogs," Anesth. Analg., vol. 2, pp. 325-331, 1998.

[10] D. Kouamé, Modélisation paramétrique et détection de rupture en traitement du signal ultrasonore, Ph.D. thesis, university of Tours, France, 1996.

[11] F. Wendling, S.A. Jones, and D.P. Giddens, "Simulation of doppler ultrasound signals for a laminar, pulsatile, nonuniform Hlow," Ultrasound Med. Biol., vol. 18, pp. 179-193, 1992.

[12] J.L. Smith, D.H. Evans, L. Fan, P.R.F. Bell, and A.R. Naylor, "Differentiation between emboli and artefacts using dual-gated transcranial doppler ultrasound," Ultrasound Med. Biol., vol. 22, pp. 1031-1036, 1996.

[13] M.A. Moehring and J.R. Keppler, "Pulse doppler ultrasound detection characterization and size estimation of emboli in flowing blood," IEEE Trans. on Biomedical Engineering, vol. 41, pp. 35-44, 1994.

[14] M.P. Spencer, Detection of Cerebral Arterial Emboli, chapter 19, In D.W. Newell and R. Aaslid, eds. Transcranial Doppler. New York, NY: Raven Press Publishers 216-230, 1992.

[15] E. Wigner, "On the quantum correction for thermodynamic equilibrium," Phys. Rev., vol. 40, pp. 740-759, 1932.

[16] J. Ville, "Theorie et applications de la notion de signal analytique," Cables et Transmission 2eA, vol. 1, pp. 61-74, 1948.

[17] A. Grossman and J. Morlet, "Decomposition of hardly functions into square integrable wavelets of a constant shape," SIAM J. Math. Anal., vol. 15, pp. 723-736, 1984.

[18] M. Basseville and al, "Segmentation des signaux," Traitement du signal, vol. 9, pp. 115-147, 1992, suppl.

[19] I.V. Nikiforov, "A generalized change detection problem," IEEE Trans. Inf. Theo., vol. 41, pp. 171-187, 1995.

[20] S.M. Kay and S.L. Marple, "Spectrum analysis - a modern perspective," Proc. IEEE, vol. 69, pp. 1380-1419, 1981. 
[21] L. Ljung and S. Gunnarson, "Adaptation and tracking in system identification. a survey," Automatica, vol. 26, pp. 7-21, 199() .

[22] H. Akaike, "A new look at the statistical model identification," IEEE Trans. on Auto. Control, vol. 19, pp. 716-723, 1974.

[23] L. Ljung and T. Soderstrom, Theory and practice of recursive identification, MIT Press, 1983.

[24] J.M. Girault, F. Ossant, A. Ouahabi, D. Kouamé, and F. Patat, "Time-varying autoregressive spectral estimation for ultrasound attenuation in tissue characterization," IEEE Trans. UFFC, vol. 45, pp. 650-659, 1998.

[25] D. Kouamé, J.P. Remenieras, J.F. Roux, A. Ouahabi, M. Lethiecq, and F. Patat, "Effect of the compensation in an abrupt change detection problem," in IEEE Digital Signal Processing Workshop Loen Norway, 1996.

[26] D. Kouamé, J.M. Girault, J.P. Chemla, A. Ouahabi, and M. Lethiecq, "Emboli detection by parametric doppler signal processing : A preliminary study," Innov. Techn. Biol. Med., vol. 19, pp. 243-248, 1998.

[27] T. Hagglund, New estimation techniques for adaptive control, Ph.D. thesis, Dept of Automatic Control, Lund University, Sweden, 1983.

[28] J.M. Girault, Estimation de la fréquence Doppler instantanée et détection de micro-emboles, Ph.D. thesis, University of Tours, France, 1999.

[29] D. W. Droste and E.B. Ringelstein, "Detection of high intensity transient signals (hits): How and why ?," Eur. J. Ultrasound, vol. 7, pp. 23-29, 1998 .

[30] D. Kouamé, J.F. Roux, and A. Ouahabi, "Parametric estimation: Improvement of the rls approach using a differential approach," International J. of Modelling and Simulation, vol. 19, pp. 18-26, 1999.

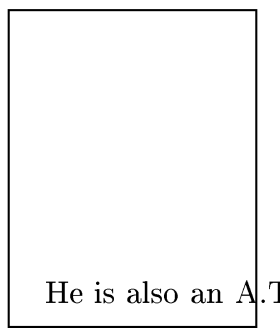

Girault Jean-Marc (S'98) was born in 1969 in Mantes - la - Jolie, France. He received the Maitrise of Electrical Engineering and the D. E. A. degree of Signal processing, Biological and Medical Imaging (SIBM) in 1996 from Angers University, France. He currently is working toward the Ph. D. degree in Sciences de l'ingénieur from Tours University, G.I.P. Ultrasons - L.U.S.S.I..

His research area concerns signal processing in biological tissues characterization, embolus detection and Doppler signal processing. He is an IEEE Student Member.

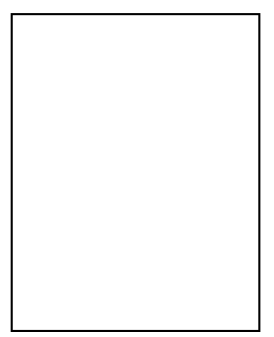

Kouamé Denis (M’98) was born in 1967 in Cote d'Ivoire. He graduated in Computer Integrated in Manufacturing Engineering from the Ecole d'Ingénieur de Tours in 1992. He received the D.E.A. degree in Automatic - Control and Computer Engineering from the University of Tours, France followed by the Ph. D. degree in Computer Engineering and Automatic - Control and Signal Processing from the University of Tours, France in 1996. Since 1994 he is with the G.I.P. Ultrasons - L.U.S.S.I. He teaches in the Tours university as an

assistant professor. 
His research interests include sensor signal processing, modeling, parameter estimation, change detection, and Doppler processing and their industrial and biomedical applications.

Ouahabi Abdeldjalil (M'98) graduated in electrical engineering from the Ecole Polytechnique of Algiers in 1979 and received thr Ph. D degree and the Doctorat d'Etat of signal processing from the Institut National Polytechnique of Grenoble, France, in 1983 and 1992, respectively.

From October 1983 to 1990, he was with the University of Science and technology of Algiers, and frpm December 1990 to September 1994, he was the Director of the Institut of Electronics of Algiers. In 1994, he joined l'Université du Maine, Le Mans, France, as a Visiting Professor of electronic and computer engineering. He is a Professor of signal processing at the University of Tours, France. His research interests are in digital signal processing, spectral analysis, time-frequency analysis, wavelet transforms, and their applications to communications, speech processing, biomedical, and ultrasonic techniques. He is the author of more than 40 journals and conference papers and five textbooks.

Dr. Ouahabi was the general Chairman of the International Conference of Signals and System (I.C.S.S.'94) and Chairman of the special session of the IEEE international Conference of Electronics, circuits and systems (I.C.E.C.'99).

Patat Frédéric was born in Lyon, France, in 1958. He received an engineering degree from the Ecole Polytechnique in 1980, the Doctorat in Physical Acoustics in 1984 and became a medical doctor in 1991. He is a teacher of biophysics as an Assistant Professor at the Medical Faculty of Tours, and he is a member of the staff of the Nuclear Medecine and Ultrasound Department at Tours Hospital.

Dr. Patat's intłrest is focused on biomedical instrumentation and acoustics within L.U.S.S.I. - G.I.P. Ultrasons at the University of Tours. 
List OF TABLES

I

List OF TABLES 
TABLE T

\begin{tabular}{|l|c|c|c|c|c|}
\hline \multirow{2}{*}{ Methods } & \multicolumn{2}{|c|}{ Non Parametric } & \multicolumn{2}{c|}{ Parametric } \\
\cline { 2 - 6 } & $D I_{1}$ & $D I_{2}$ & $D I_{3}$ & $D I_{4}$ & $D I D_{5}$ \\
\hline$E B R_{\text {min }}(\mathrm{dB})$ & 12 & 10 & 10 & 4 & 5 \\
\hline
\end{tabular}

$E B R_{\text {min }}$ obtained from PFA $=P N D \leq 10 \%$ and for all methods evaluated from artificial signals. $E B R_{\min }$ corresponds to a reliable detection of embolus. 


\section{List OF FiguRES}

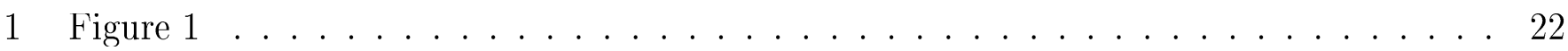

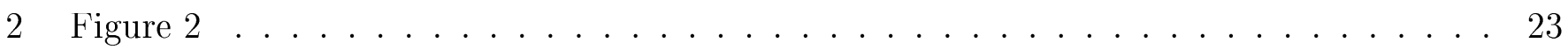

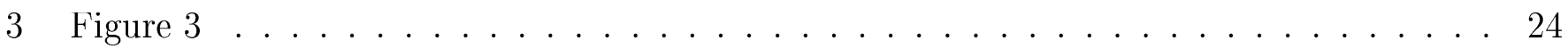

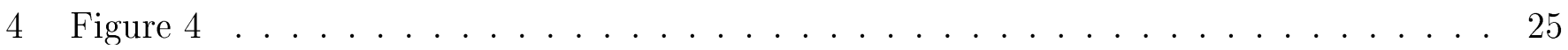

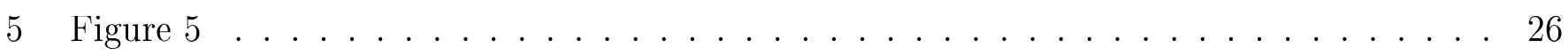

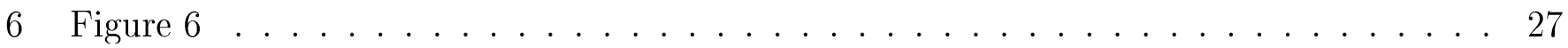

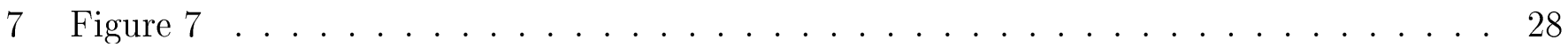

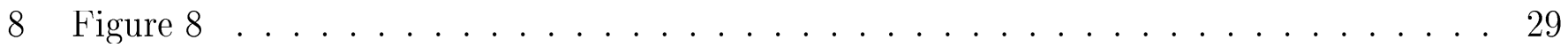

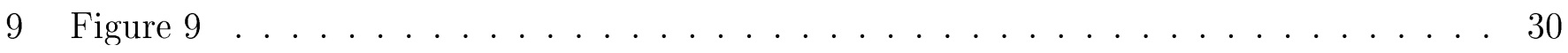

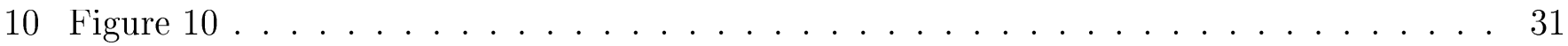

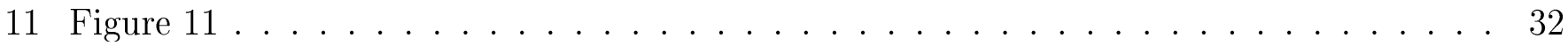




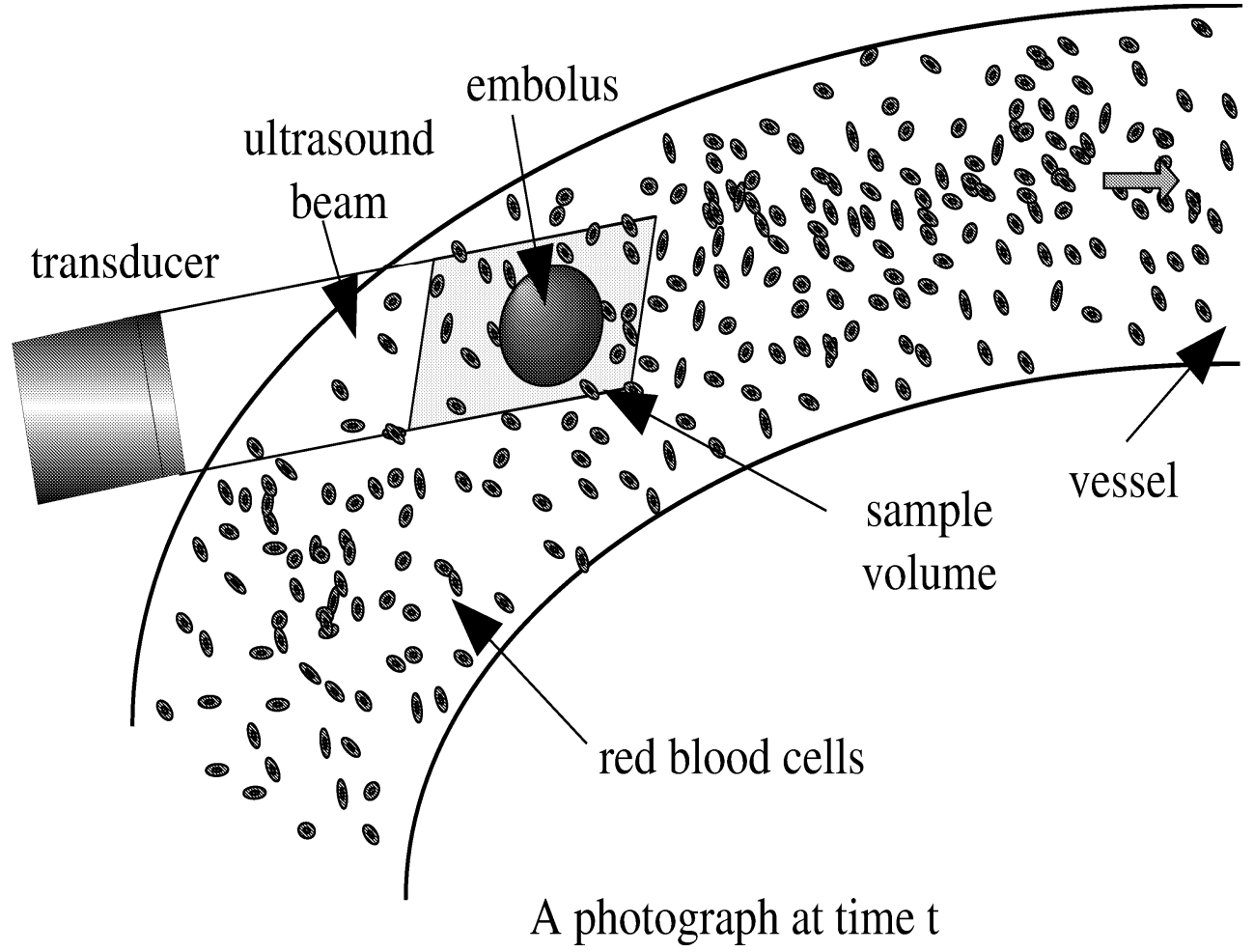

Fig. 1.

An overscheme of a large embolus crossing the sample volume in a blood vessel. 


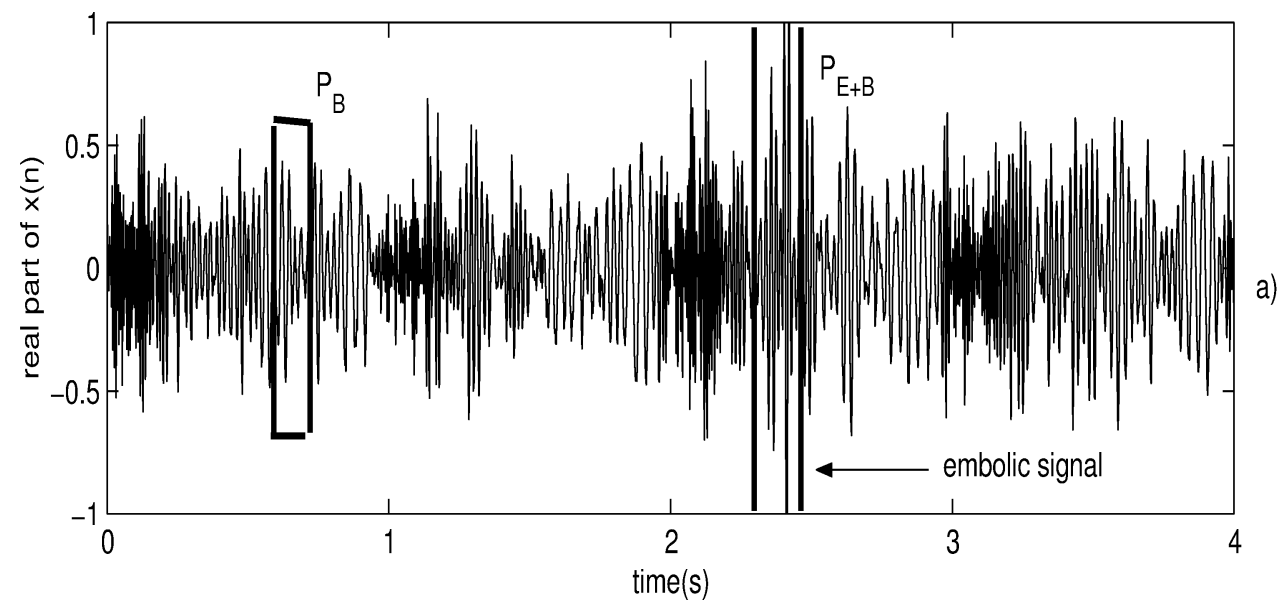

a)

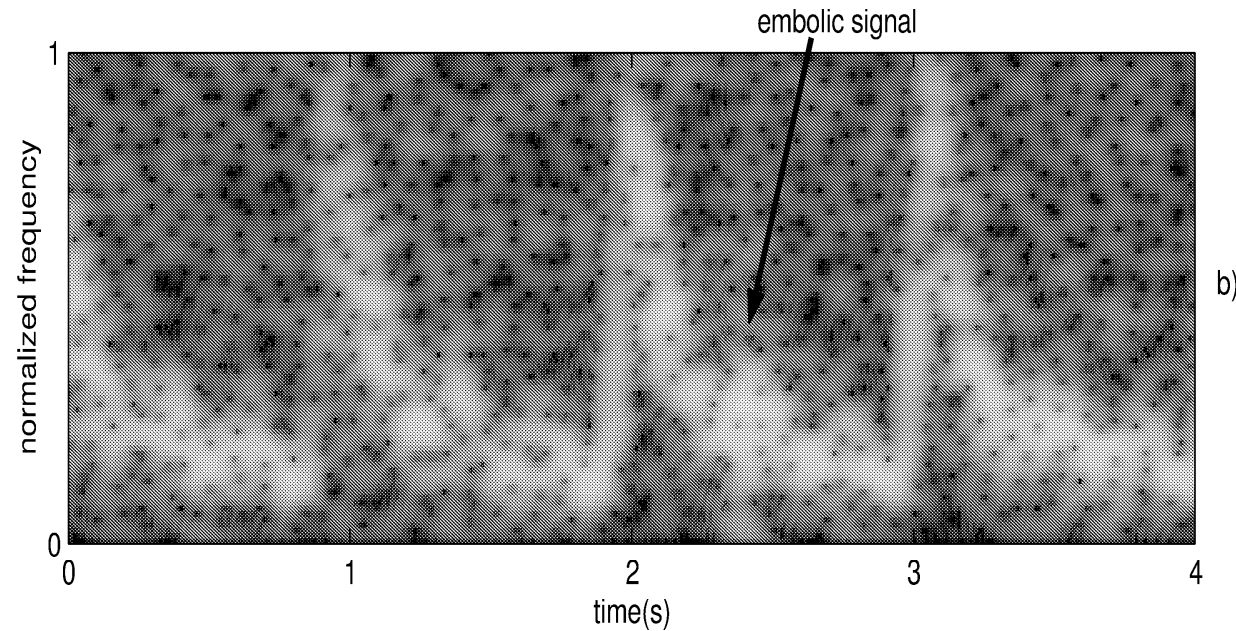

Fig. 2.

A time-frequency representation of the blood velocity in a vessel with an embolus $(E B R=6 d B)$. An artificial Doppler signal $x(n)$ a) and its spectrogram b). A high intensity associated to an embolus appears in the time-frequency domain with a poor resolution. 


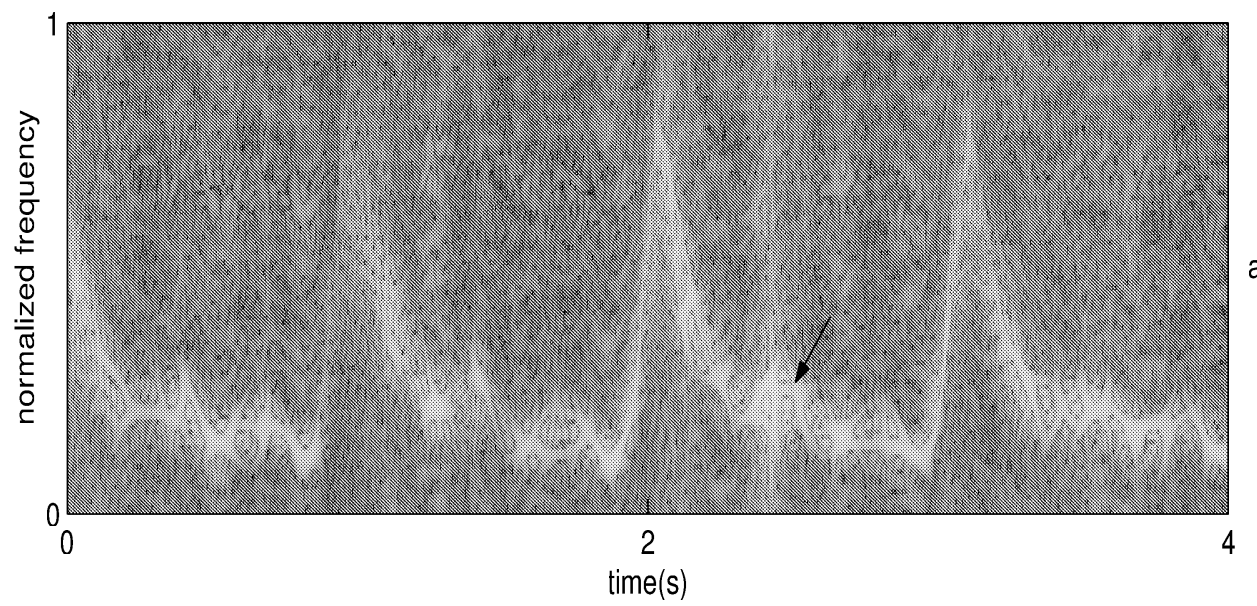

a)

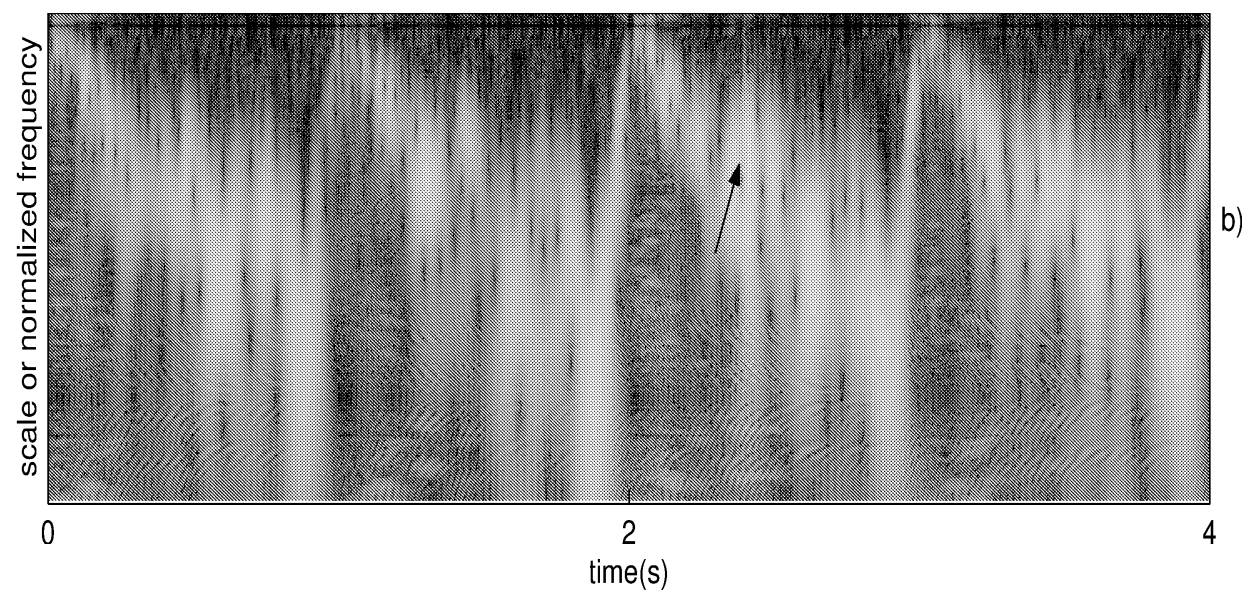

Fig. 3.

A time-frequency representation of the velocity profile of blood circulating in a vessel with an embolus $(E B R=6 d B)$. A time-frequency representation evaluated by the Wigner-Ville a) and Wavelet $b)$ transforms of an artificial Doppler signal. A high intensity associated to an embolic signal appears with different resolutions for each method. 

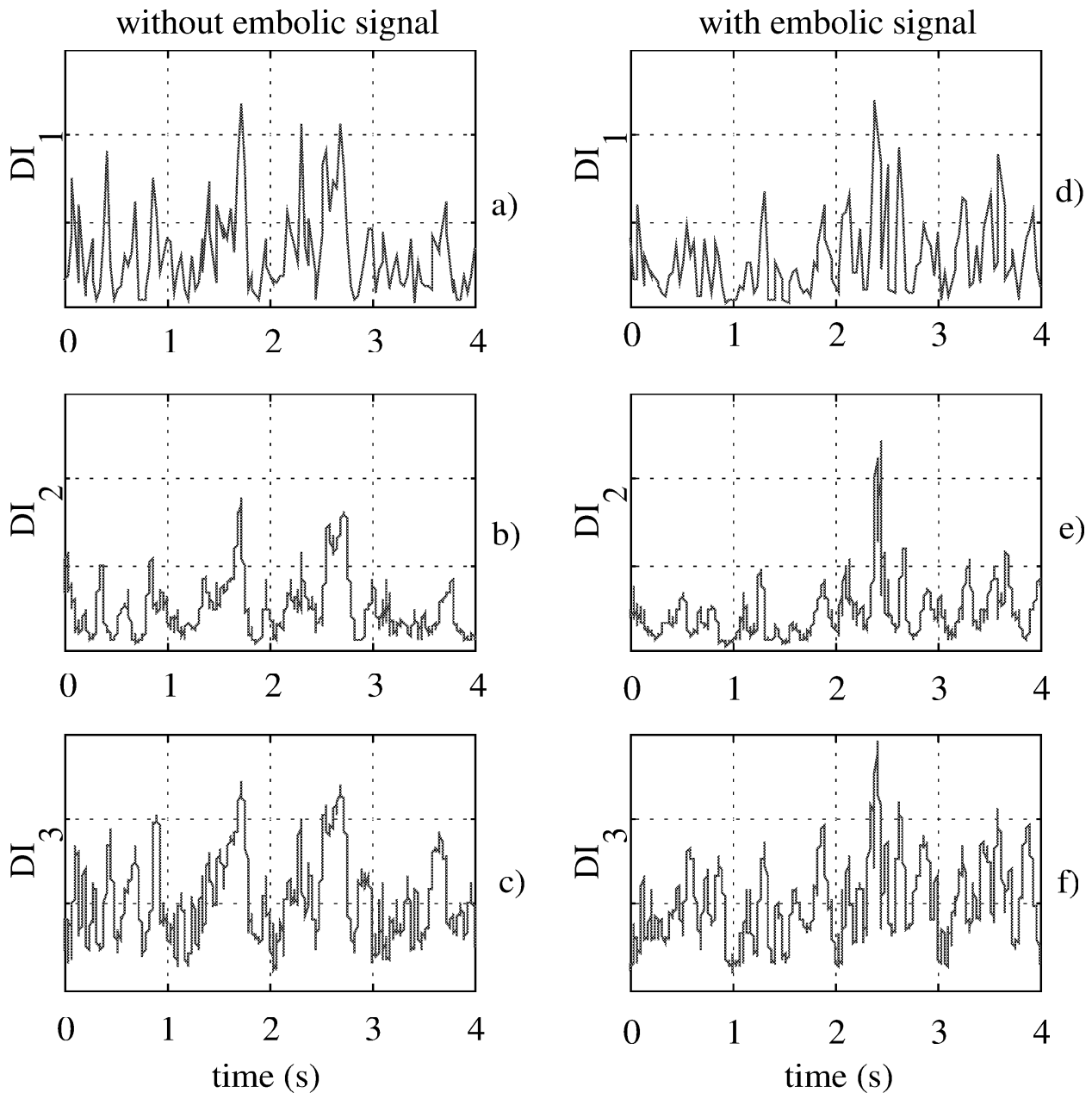

Fig. 4.

Decision Information (DI) of the non parametric methods. Fourier a), WV b) and WT c) approaches without embolic signal. Fourier d), $W V$ e) and $W T f)$ approaches with embolic signal $(E B R=6 d B)$. All three of the DI point out a high amplitude where there is an embolic signal. 

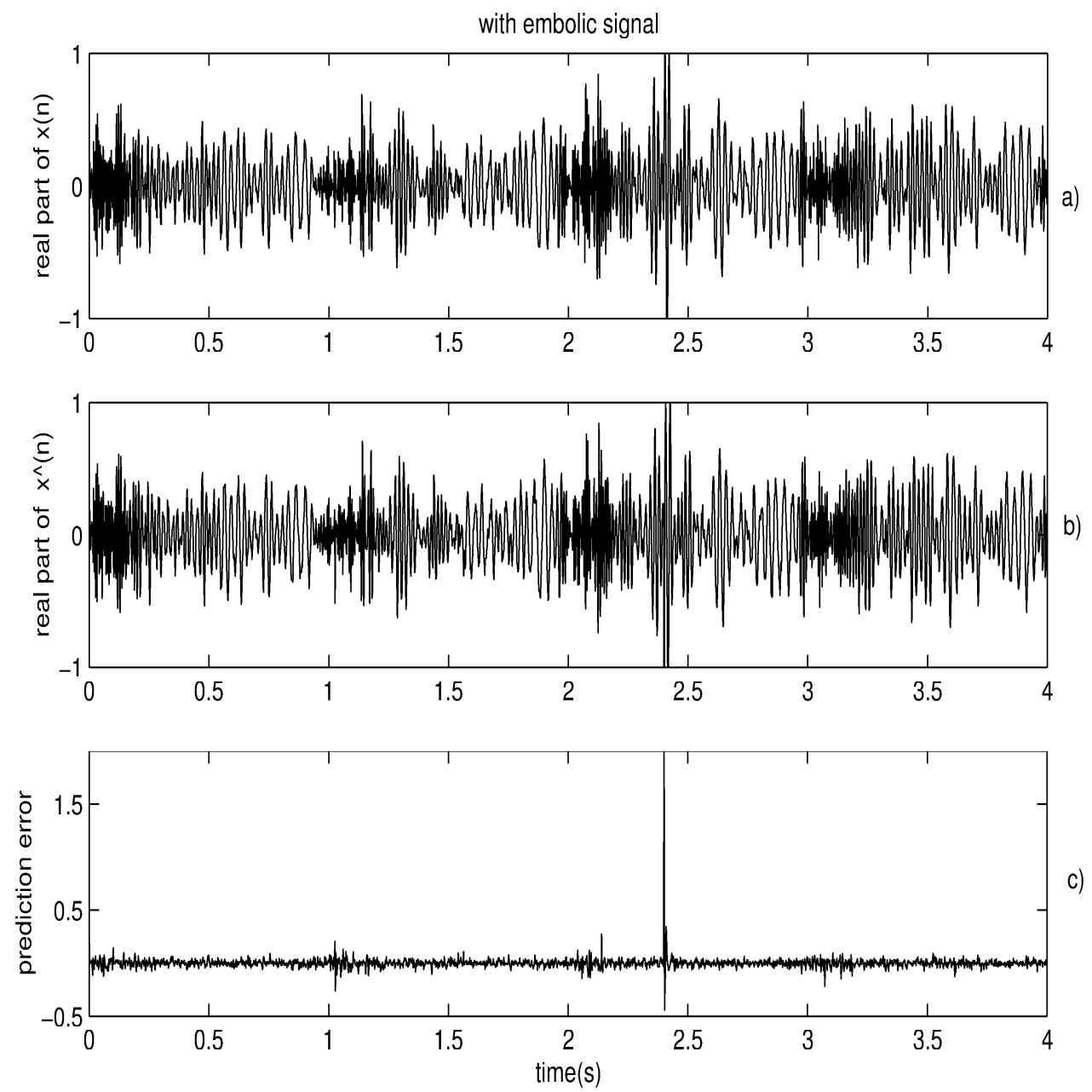

Fig. 5.

Prediction error of a synthetic signal. Real part of a Doppler signal backscattered by blood plus an embolic signal $(E B R=6 d B)$ a), real part of the Doppler signal modeled by an AR filter b) and error between the Doppler signal and its modeled signal c). 

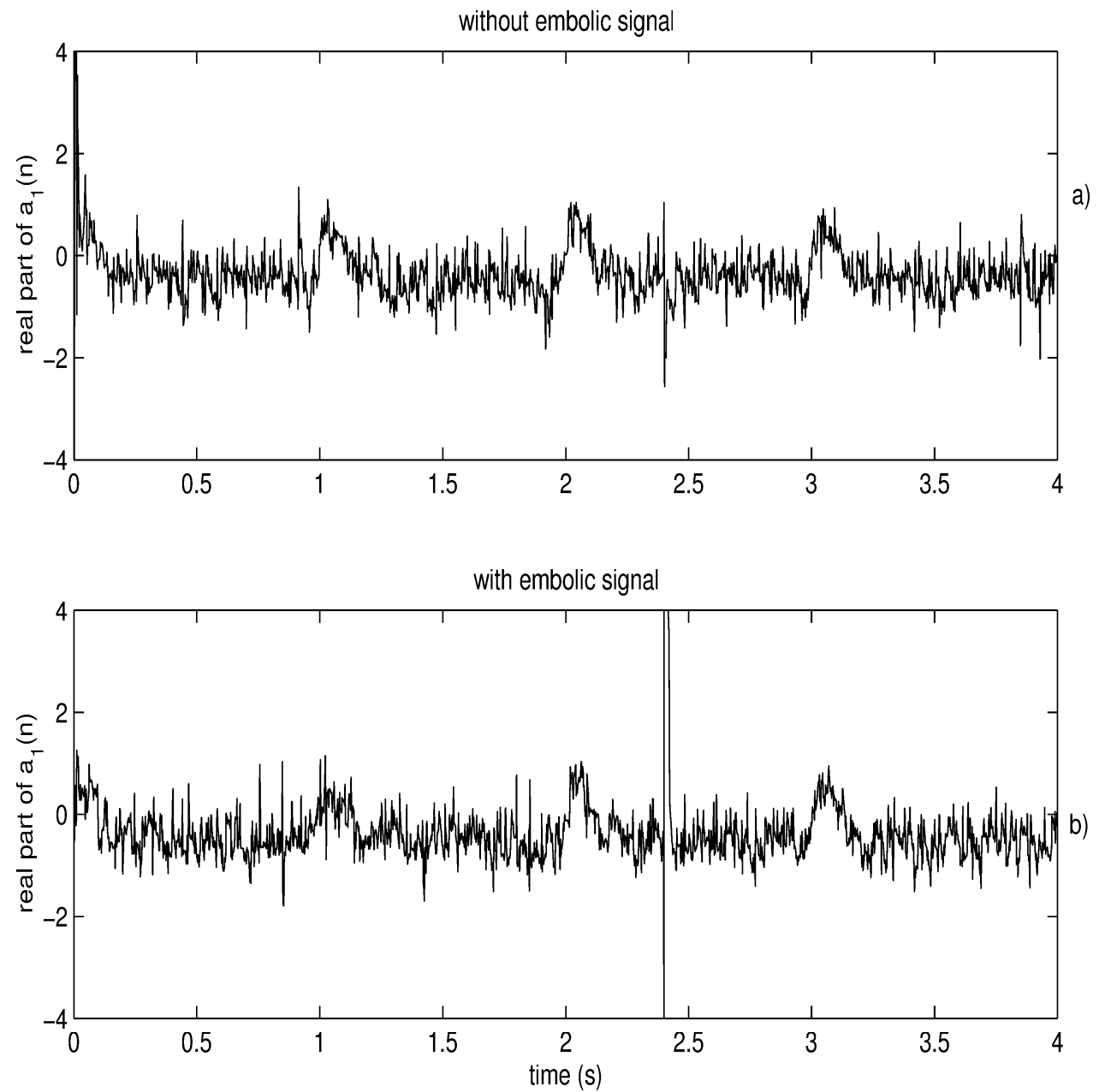

Fig. 6.

AR parameters of the parametric model. Real part of the autoregressive parameter $a_{1}$ without an embolic signal a) and with an embolic signal $(E B R=6 d B) b)$. Slow variations are related to the systole and diastole phases of the circulating blood. 

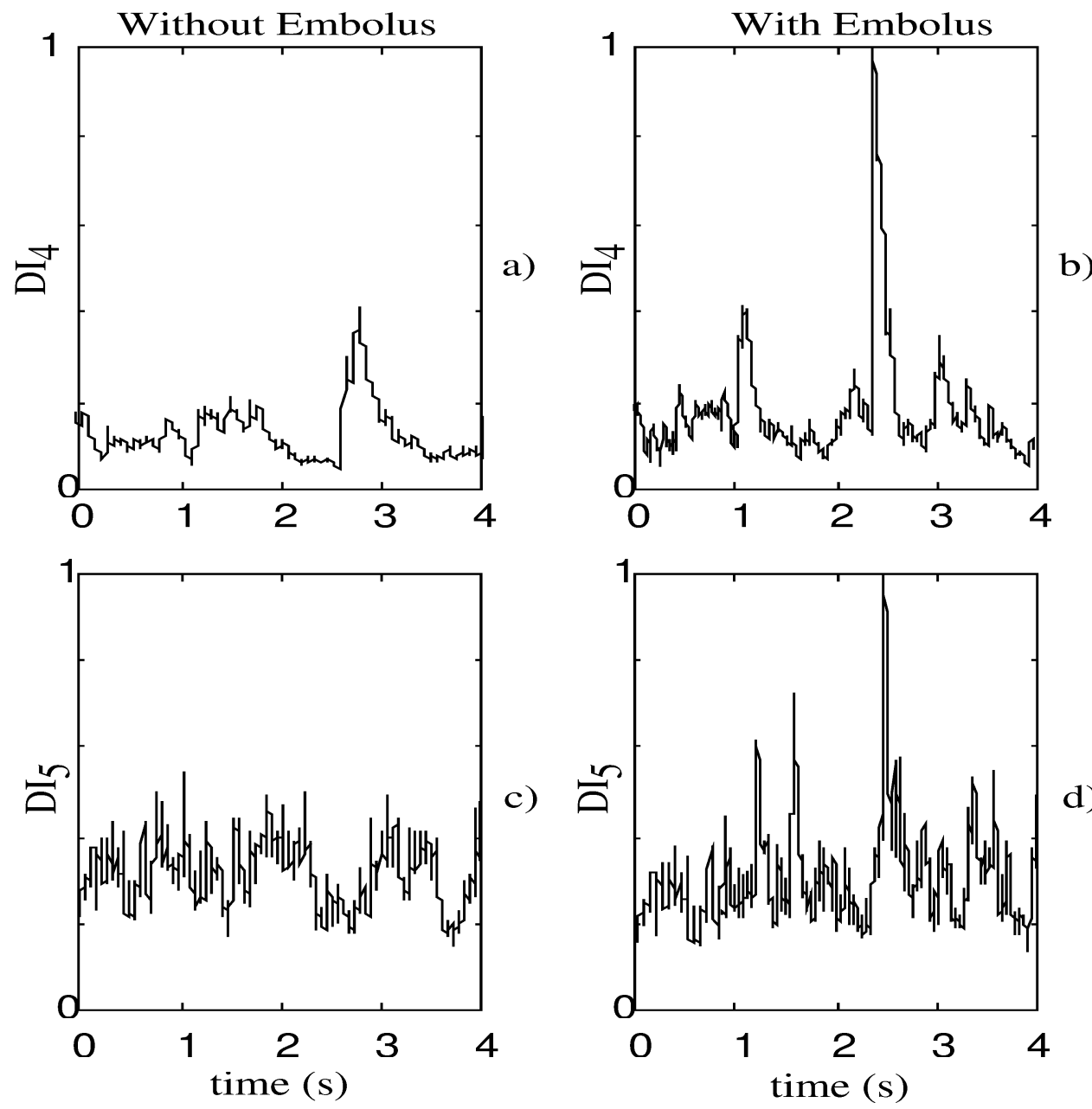

Fig. 7.

Decision Information (DI) of the parametric methods. DI $I_{4}$ without embolic signal in a), a convergence region is located at the beginning of $D I_{4}$ and $D I_{5} . D I_{4}$ with an embolic signal $(E B R=6 d B)$ in $b)$, the embolus signature is located at about $2.5 s . D I_{5}$ without an embolic signal in c), the numerical derivation explains the stochastic nature of $D I_{5} . D I_{5}$ with embolic signal in d). 

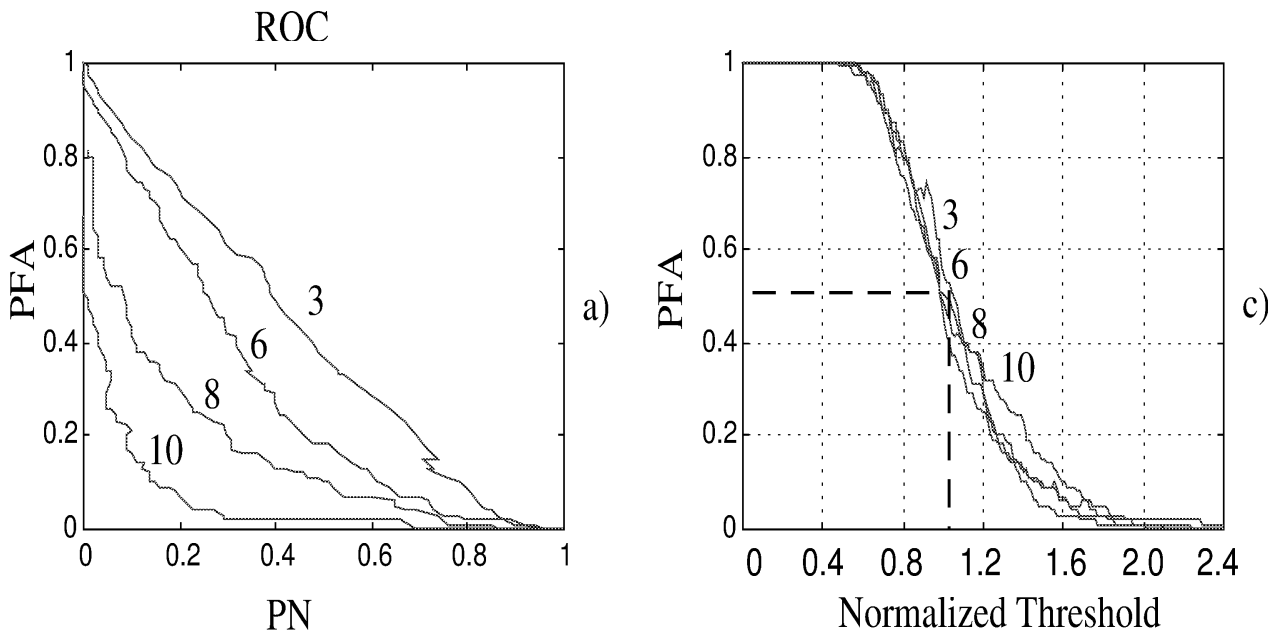

D
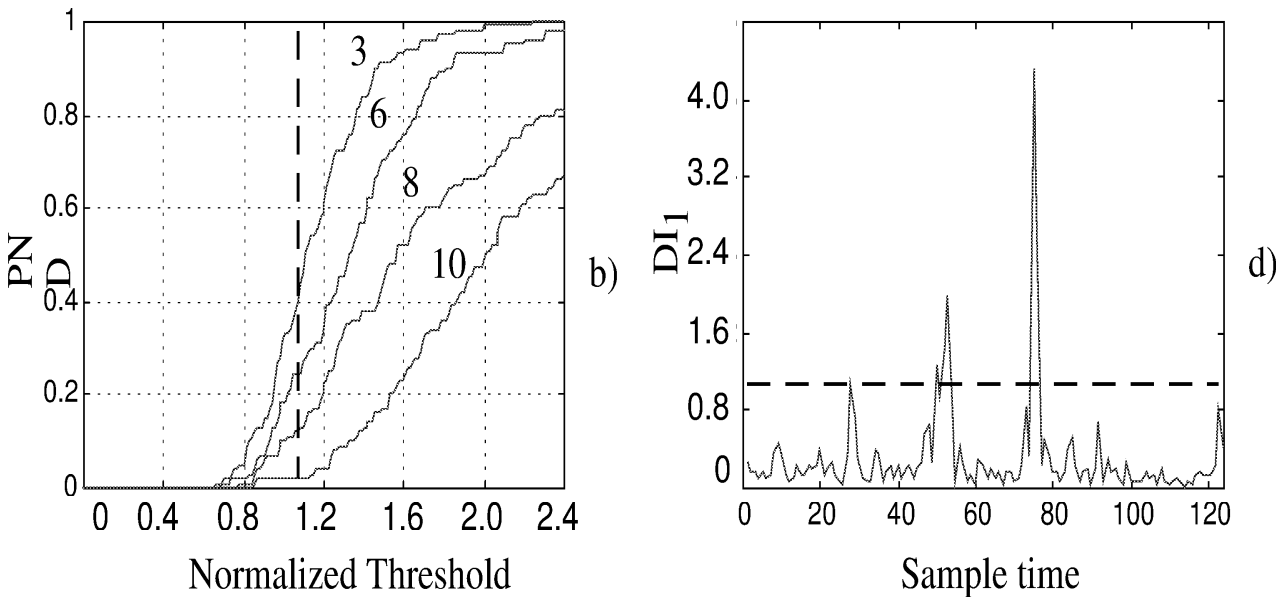

Fig. 8.

ROC curves showing the PFA versus PND. Each curve corresponds to different values of EBR a). PND versus threshold b). PFA versus threshold $c$ ). DI in the case of one embolus in the sample volume and for $E B R=6 d B$. 

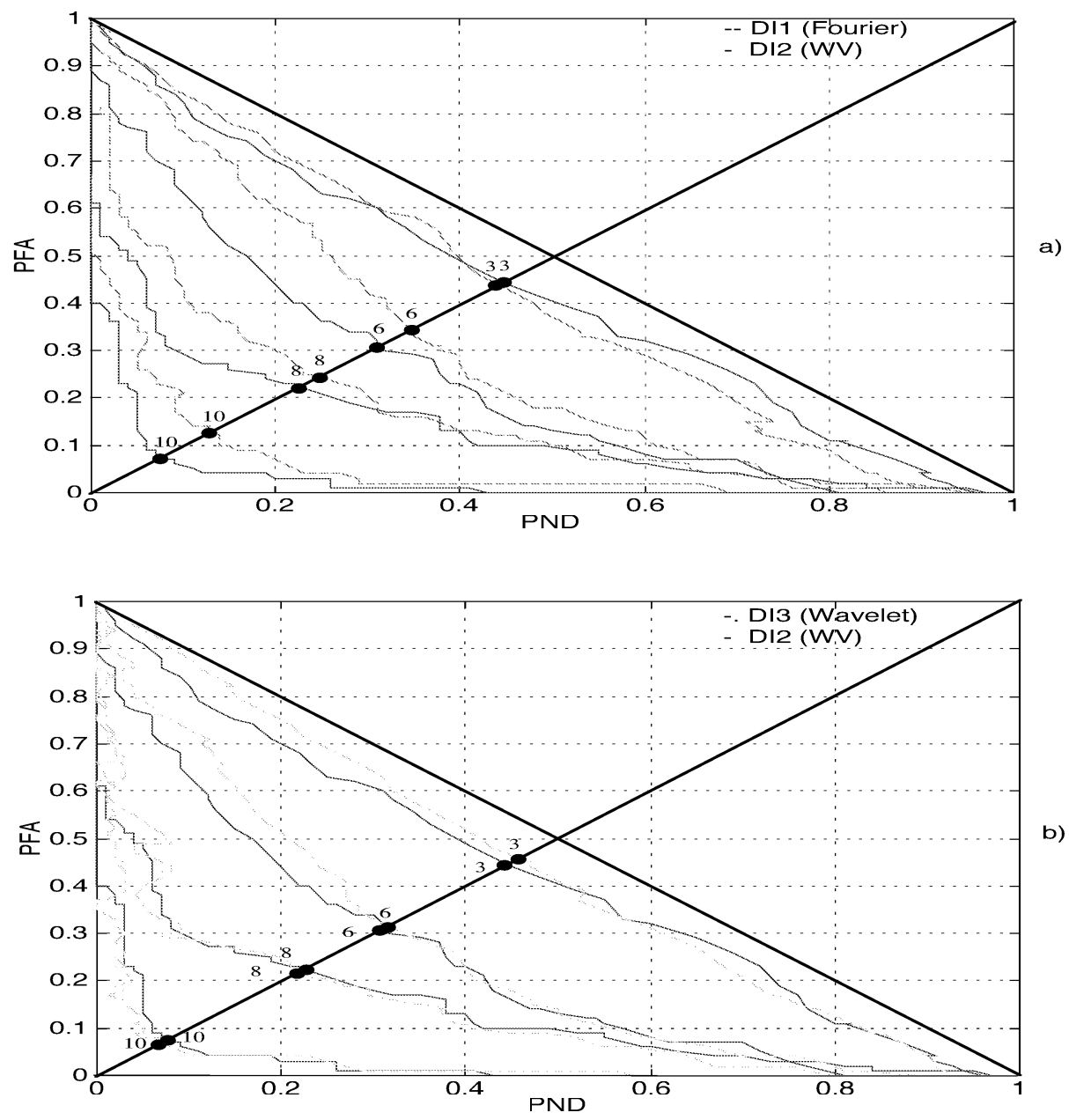

Fig. 9 .

Non parametric ROC curves for different values of EBR. DI $I_{1}$ (Fourier) (dotted line) and DI $I_{2}$ (WVT) (dark line) a) and $D I_{3}(W T)$ (dashed dot line) b). When no embolic signal is detected, curves tend to a straight line that passes through the point $(0,1)$ and $(1,0)$. For ideal methods, curves tend to the point $(0,0)$. 

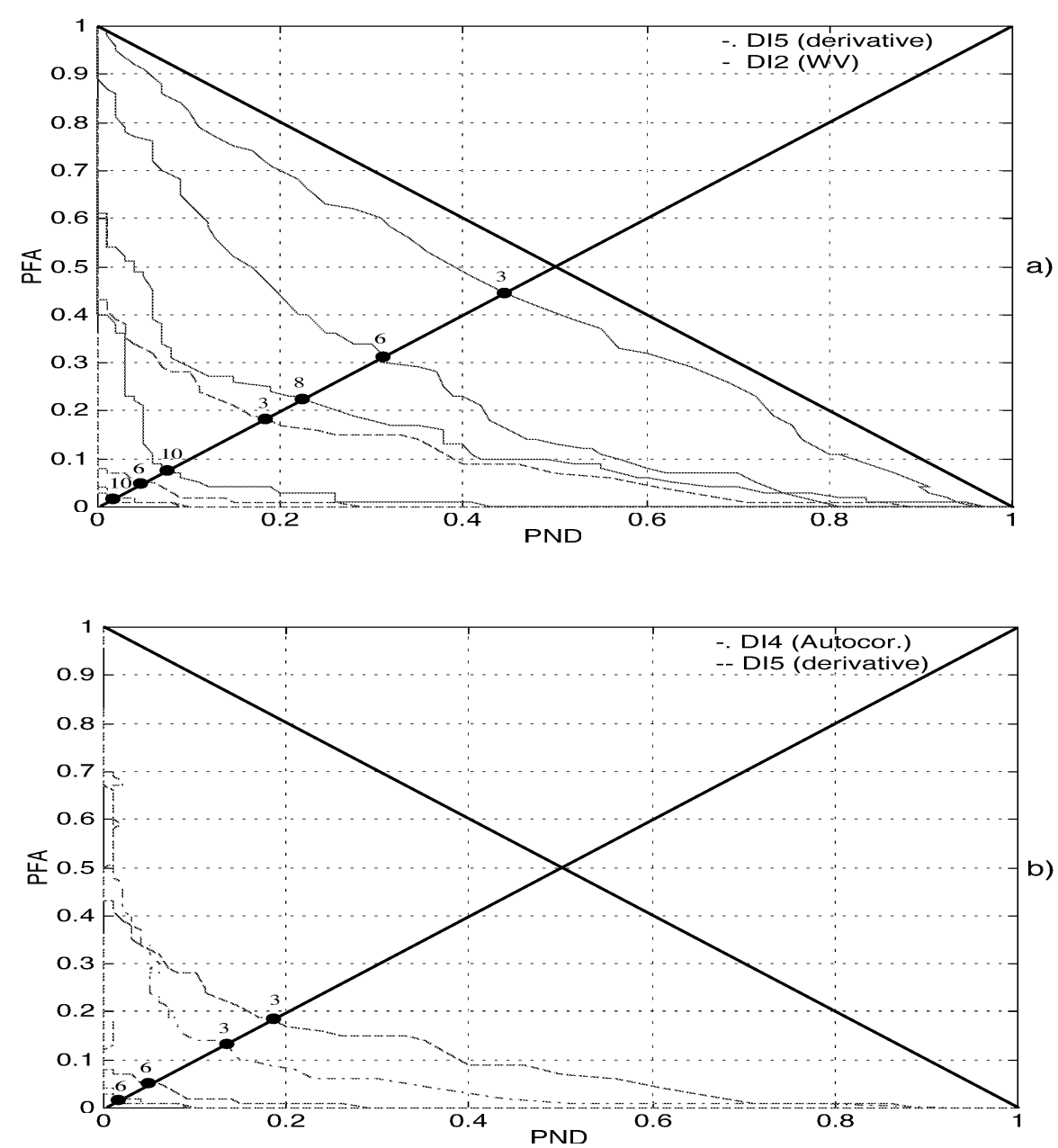

Fig. 10.

ROC curves for different value of EBR with different DI. DI $I_{2}$ (dark line) and $D I_{5}$ a) respectively correspond to the Wigner-Ville and the derivative of AR parameters approaches. DI $I_{4}$ (dashed dot line) b) corresponds to the method of innovation autocorrelation. When no embolic signal is detected, curves tend to a straight line that passes through the point $(0,1)$ and $(1,0)$. For ideal methods, curves tend to the point $(0,0)$. 


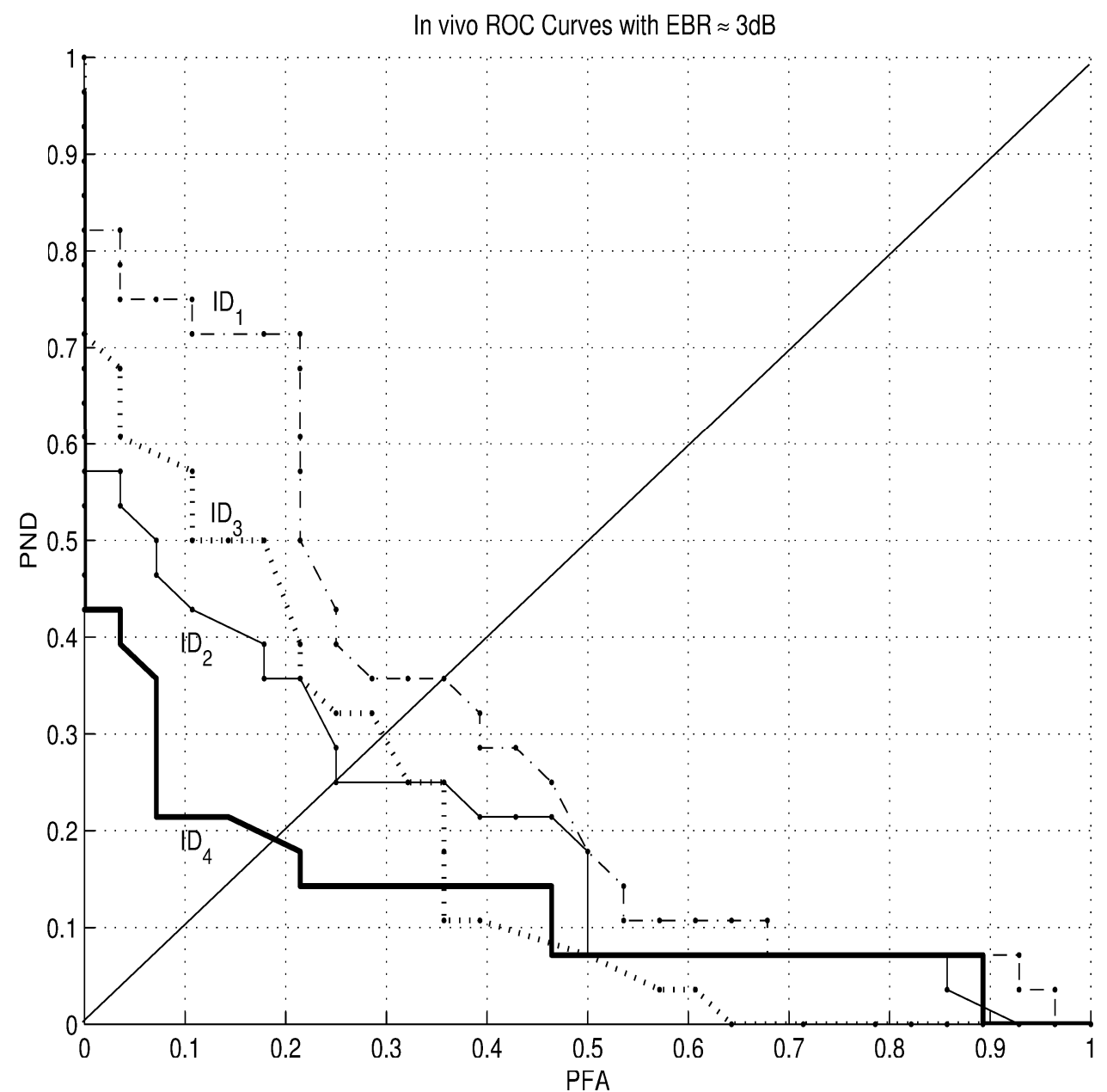

Fig. 11.

$R O C$ curves from in vivo study and with different $D I$. 